\title{
Oral immunotherapy for treatment of peanut allergy
}

\author{
Joan H Dunlop
}

Pediatrics, Johns Hopkins University School of Medicine, Baltimore, Maryland, USA

\section{Correspondence to} Dr Joan H Dunlop, Pediatrics, Johns Hopkins University School of Medicine, Baltimore, MD 21287, USA: jdunlop7@jhmi.edu

Accepted 17 June 2020 Published Online First 14 July 2020

\section{Check for updates}

(C) American Federation for Medical Research 2020. No commercial re-use. See rights and permissions. Published by BMJ.

To cite: Dunlop JH. $J$ Investig Med 2020;68:1152-1155.

\begin{abstract}
The US Food and Drug Administration's approval of a peanut oral immunotherapy product in January 2020 is a landmark development in the field of food allergy therapy. While food allergy prevalence has been increasing, this product is the first approved therapy for food allergy. Oral immunotherapy has many similarities to subcutaneous immunotherapy and drug desensitization protocols, but does not lead to sustained unresponsiveness. The studies leading to approval of the Palforzia product demonstrated increase in the amount of peanut protein able to be consumed, with $67 \%$ of subjects randomized to the treatment arm able to consume $600 \mathrm{mg}$ of peanut protein in double-blind placebo-controlled food challenge at study exit. However, side effects are an important consideration, and dropout rates in studies of Palforzia ranged from $11 \%$ to $21 \%$. Postmarketing surveillance of this product will be critical in assessing its long-term risks and benefits.
\end{abstract}

\section{INTRODUCTION}

Food allergy is a growing problem in the USA and worldwide, currently affecting 5\%-10\% of the population of the developed world. ${ }^{1}$ In the USA, peanut allergy is the most prevalent food allergy, affecting 1 in 70 children, the majority of whom do not outgrow this allergy. ${ }^{2}$ Patients with any food allergy can develop lifethreatening anaphylaxis on ingestion of even small amounts of the offending allergen, and allergic reactions account for 300,000 emergency visits in the USA annually. ${ }^{3}$ To prevent and treat these reactions, patients and their families must adhere to a careful regimen of allergen avoidance and carry injectable epinephrine for treatment of anaphylaxis at all times. Despite these precautions, accidental exposures and subsequent reactions can and do occur, with estimates of annual incidence ranging from $10 \%$ to $15 \%$ among peanut allergic children. ${ }^{4}$ This need for constant vigilance poses a significant burden, and studies have demonstrated that quality of life among those with food allergy is significantly impaired. Because food allergy is currently a lifelong disease without a cure, this creates a long-standing societal disease burden. ${ }^{5}$ Peanut allergy in particular has a low rate of being outgrown, making it a good candidate for initial therapeutic trials. ${ }^{6}$
The first Food and Drug Administration (FDA)-approved therapy to treat food allergy, Palforzia, was approved by the US FDA in January 2020 to treat peanut allergic children 4-17 years of age. ${ }^{7}$ In this review, we will discuss the history of oral immunotherapy (OIT) as a therapeutic modality for food allergy, understand the current limitations of food allergy research and therapies, and summarize the major studies of peanut OIT leading to the approval of Palforzia. As a review of existing literature, ethics approval was not required for this manuscript.

OIT has its roots in subcutaneous immunotherapy (SCIT), known colloquially as 'allergy shots', a cornerstone of the practice of allergists. SCIT is typically used for the treatment of allergic rhinitis and allergic conjunctivitis among those sensitized to environmental allergens, both perennial and seasonal. SCIT involves the subcutaneous injection of gradually increasing quantities of the allergenic substance to which the patient is sensitized until a maintenance dose is reached, then continuing to administer the maintenance dose over the course of several years, allowing the patient to eventually develop longlasting tolerance to the allergen, and amelioration of allergic rhinitis, conjunctivitis and asthma symptoms in the majority of patients. ${ }^{8}$

In a similar vein, temporary desensitization to medications is used by allergists for the treatment of drug allergy when no adequate alternative exists for the allergenic drug, most prototypically penicillin. While there exist many similarities between drug desensitization and SCIT, they differ in that drug desensitization offers temporary ability to tolerate the offending drug. Once the drug is discontinued, the patient is again allergic to the drug, and will demonstrate anaphylaxis if the drug is reintroduced. ${ }^{9}$

\section{TERMINOLOGY}

To differentiate between these 2 endpoints, temporary and long-term desensitization, the long-term non-reactiveness to allergic stimuli has been deemed 'sustained unresponsiveness' whereas the temporary increase in the threshold for reactivity to an allergenic substance is termed 'desensitization'. ${ }^{10}$ Maintenance of the desensitized state requires continued exposure to the allergen in question. ${ }^{11}$ 


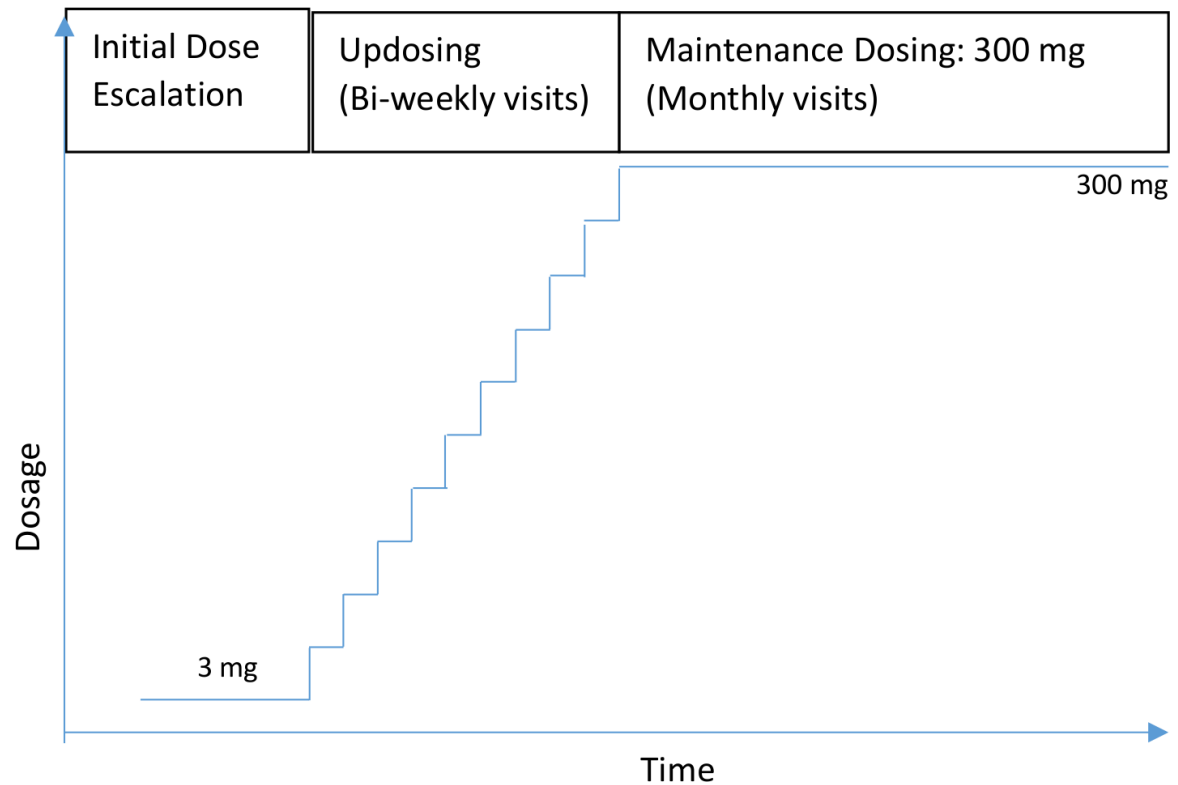

Figure 1 Dosing strategy for oral immunotherapy (OIT).

Both desensitization and immunotherapy protocols typically begin with small amounts of the allergenic substance, with slow increase in the dose, called an 'up-dosing period', until a 'maintenance' dose is reached. There are varying intervals of time used for the up-dosing period, with rush or compressed dosing protocols allowing for rapid increase of the dose in the office or hospital setting (figure 1).

\section{HISTORY}

Naturally, when faced with the burgeoning problem of food allergy, allergists attempted to use a long-known therapeutic modality, SCIT, for its treatment. The first studies used subcutaneous peanut extract, and were able to demonstrate desensitization. However, all subjects experienced systemic reactions during the up-dosing and with maintenance dosing. Thus, this modality was deemed infeasible. ${ }^{28}$

While reports of OIT date back to the beginning of the 20th century, Patriarca et al first demonstrated the successful treatment of allergies to cow's milk, egg, fish, and fruits with standardized OIT protocols in the 1980s and 1990s, setting the stage for clinical trials of OIT. ${ }^{12}$ The first proof of concept OIT study for the treatment of food allergy was conducted in 2007 with egg allergy as its therapeutic target. A proof of concept OIT study targeting peanut allergy was published in 2009, using defatted peanut flour, a form of the allergen still used today in peanut OIT. ${ }^{13}$ The defatted powder is more stable when stored, and the defatting is also reported to make the product more palatable to patients. ${ }^{14}$

\section{SIDE EFFECTS OF OIT}

The greatest risk of OIT, as for SCIT, is acute allergic events related to dosing. The symptoms of an allergic reaction can range from mild nasal congestion or gastrointestinal (GI) discomfort to systemic anaphylaxis (table 1). A meta-analysis of adverse events in peanut OIT studies was published in 2019. The analysis included 12 peanut OIT studies. Overall, the authors concluded that peanut OIT could successfully desensitize subjects to peanut (risk ratio
(RR) 12.42, 95\% CI 6.82 to 22.61), but subjects using peanut OIT were significantly more likely to experience more episodes of anaphylaxis when compared with those subjects practicing strict avoidance (RR 3.12), regardless of phase of treatment (ie, build-up vs maintenance). ${ }^{15} \mathrm{~A}$ broader analysis of peanut OIT side effects, which included non-blinded studies, concluded that the overall risk of dropping out of the study due to side effects was $6.6 \%$, and the risk of an adverse event requiring epinephrine use was $7.6 \% .^{16}$

Table 1 Adverse events affecting more than $5 \%$ of subjects in phase 3 clinical trial

\begin{tabular}{|c|c|c|}
\hline Adverse event & Treatment group (\%) & Placebo group (\%) \\
\hline Abdominal pain & 52.2 & 24.2 \\
\hline Vomiting & 41.4 & 24.2 \\
\hline Pruritus & 41.1 & 27.4 \\
\hline Upper abdominal pain & 40.9 & 21.0 \\
\hline Cough & 40.9 & 33.9 \\
\hline Throat irritation & 40.9 & 27.4 \\
\hline Oral pruritus & 40.6 & 16.1 \\
\hline Nausea & 39.2 & 23.4 \\
\hline Urticaria & 38.4 & 24.2 \\
\hline Rhinorrhea & 30.4 & 22.5 \\
\hline Sneezing & 26.3 & 14.5 \\
\hline Throat tightness & 23.1 & 6.5 \\
\hline Rash & 21.8 & 14.5 \\
\hline Oral paresthesia & 17.5 & 6.5 \\
\hline Systemic allergic reaction & 14.2 & 3.2 \\
\hline Lip swelling & 10.2 & 4.0 \\
\hline Dyspnea & 11.8 & 4.0 \\
\hline Dysphonia & 6.7 & 1.6 \\
\hline Ear pruritus & 6.7 & 0 \\
\hline Chest discomfort & 6.5 & 0.8 \\
\hline
\end{tabular}

Adapted from Vickery et al [14]. 
A concerning adverse effect of OIT is eosinophilic esophagitis (EOE), which is a non-IgE-mediated disease characterized by eosinophilic infiltration of the esophagus. Symptoms include nausea, odynophagia, and food impaction. Diagnosis is dependent on endoscopically obtained biopsy specimens and long-term complications of EOE include esophageal strictures requiring dilation. In previous OIT studies, EOE has occurred in 2\%-5\% of subjects and is thought to resolve with cessation of OIT administration. Food allergy is a risk factor for EOE, and some researchers speculate that a large portion of patients undergoing OIT may have pre-existing esophageal eosinophilia. ${ }^{2} 1718 \mathrm{~A}$ major challenge of monitoring the development of EOE in patients undergoing OIT is the invasive means necessary for diagnosis, and thus this may potentially be an underdiagnosed side effect of OIT.

\section{UNIQUE CHALLENGES OF FOOD ALLERGY RESEARCH}

One significant hurdle to food allergy research is the relative lack of specificity of food allergy testing. While serum allergen-specific $\mathrm{IgE}$ and skin prick test are sensitive for the detection of food allergy, food challenge remains the gold standard for diagnosis, with serum-specific IgE and skin prick test demonstrating specificities of approximately $80 \%$. However, food challenges are time and staff intensive, and carry with them the risk of serious allergic reaction. ${ }^{1}$

Furthermore, food allergy patients may have significant, unpredictable, intrasubject variability in the threshold dose to which they react. Therefore, the most rigorous clinical trials of OIT require double-blind placebo-controlled food challenge (DBPCFC) at study entry and conclusion, a costly and time-consuming effort that carries risk of serious allergic reaction for study subjects. ${ }^{11}$

\section{PEANUT OIT TRIALS}

Initial trials of peanut OIT used a range of starting and maintenance doses. At this point, typical peanut OIT protocols begin with $0.5 \mathrm{mg}$ of peanut protein, and reach maintenance doses ranging from 300 to $4000 \mathrm{mg}$. For reference, a typical peanut pod contains 2 peanut kernels, and each peanut kernel typically contains $250-300 \mathrm{mg}$ of peanut protein. The Palforzia product uses a maintenance dose of $300 \mathrm{mg}$ of peanut protein (figure 1).

Bird et al published the phase 2 clinical trial of peanut OIT in $2018 .{ }^{19}$ This randomized, double-blind, placebocontrolled trial included 55 patients between the ages of 4 and 26 years. This study used DBPCFCs to determine allergic status for study entry and determination of the primary endpoint, which was consumption of a cumulative dose of $443 \mathrm{mg}$ of peanut protein ( $300 \mathrm{mg}$ maximum dose). An important consideration is the $21 \%$ dropout rate among those in the treatment arm, with 4 subjects leaving due to dosing-related GI symptoms, and 2 additional subjects developing GI symptoms in combination with other symptoms leading to study dropout. Endoscopy was pursued in 1 subject, leading to a diagnosis of EOE. While no subjects received repeat endoscopy prior to study completion, all 6 subjects had demonstrated resolution of GI symptoms within 3 weeks of stopping OIT.

The phase 3 clinical trial of peanut OIT was published by Vickery et al in $2019 .{ }^{14}$ In the primary phase 3 study, the
Peanut Allergy Oral Immunotherapy Study of AR101 for Desensitization (PALISADE), DBPCFCs were used for entry and exit, in a fashion similar to the phase 2 study. However, the primary endpoint was the ability to tolerate at least the $600 \mathrm{mg}$ dose at the exit challenge, compared with $300 \mathrm{mg}$ in the phase 2 trial. The study enrolled 551 patients between the ages of 4 and 55 years, but the bulk of the patients were in the $4-17$ years old age group, and the primary outcome was an analysis of only the subjects aged $4-17$ years old. In this phase 3 study, $11.6 \%$ of subjects in the treatment arm withdrew secondary to adverse events, compared with $2.4 \%$ in the placebo arm.

In this trial, $67.2 \%$ of subjects randomized to the treatment arm tolerated a minimum of $600 \mathrm{mg}$ in exit challenge with no more than mild symptoms compared with $4 \%$ of subjects in the placebo arm. There were only 56 subjects $>18$ years of age, and the proportion meeting the $600 \mathrm{mg}$ endpoint did not statistically significantly differ between treatment and placebo groups.

\section{PALFORZIA}

The approved Palforzia drug is administered in the office in a fashion similar to that used in the phase 2 and 3 trials. Once again, the drug is a defatted peanut powder which is contained in capsules and sachets. The contents of these packages are sprinkled into a vehicle acceptable to the patient (such as applesauce or ice cream), and consumed daily. Patients are instructed to abstain from vigorous exercise for 2 hours after dosing, and to skip their daily dose if they develop significant illness.

There is no required entry or exit challenge for typical use, and all up-dosing should occur in the office under medical supervision. Patients will continue daily dosing at home between up-dosing visits, and on reaching the maintenance dose, should continue daily dosing at home indefinitely. Currently, patients and providers interested in receiving or providing Palforzia must register with a Risk Evaluation and Mitigation Strategy program as mandated by the US FDA as a condition of this approval. ${ }^{20}$

\section{EXPECTED OUTCOMES OF OIT}

It is expected that successful completion of the up-dosing phase and the continuation of maintenance dosing will provide significant protection, preventing reactions to small accidental exposures to peanut, and attenuate reactions to larger exposures, decreasing their severity.

However, the use of this medication will not do away with the need to continue avoidance of peanut products as it does not allow users to achieve sufficient desensitization to consume regular quantities of peanut products in their diet. ${ }^{21}$ It will be important to measure patient and caregiver quality of life, to see if expected improvements in quality of life are achieved. Furthermore, patients should continue to carry their epinephrine autoinjectors, perhaps even more diligently, as a meta-analysis has demonstrated that subjects participating in OIT have more episodes of anaphylaxis than those practicing strict avoidance, with no differences seen in anaphylaxis frequency when comparing the maintenance and up-dosing periods. ${ }^{15}$

Patients receiving peanut OIT will continue to be monitored, and postmarketing surveillance will provide 
much-needed information regarding long-term OIT outcomes regarding patient practices and anaphylaxis frequency in the real-world setting.

\section{CONCLUSIONS AND FUTURE DIRECTIONS}

The approval of an inaugural product for treatment of food allergy is certainly a major step in food allergy research and treatment. As discussed above, while Palforzia will provide protection against accidental ingestions of peanut protein, it will require indefinite daily dosing and is known to cause allergic reactions, even after prolonged periods on maintenance dosing, leaving significant room for improved food allergy therapies.

Other approaches in clinical trials for peanut allergy include epicutaneous immunotherapy, which appears to have decreased risk of adverse events, vaccines, and antigen-specific monoclonal antibodies. ${ }^{22}$ The use of the OIT approach for other food allergens is also under clinical investigation, and clinicians and investigators should expect ongoing developments in the field of food allergy therapies over the coming years.

Contributors JD is responsible for the design of the work, the analysis of data, and drafting the work.

Funding The authors have not declared a specific grant for this research from any funding agency in the public, commercial or not-for-profit sectors.

Competing interests None declared.

Patient consent for publication Not required.

Provenance and peer review Commissioned; internally peer reviewed.

\section{ORCID ID}

Joan H Dunlop http://orcid.org/0000-0001-7768-8243

\section{REFERENCES}

1 Dunlop JH, Keet CA. Epidemiology of food allergy. Immunol Allergy Clin North Am 2018;38:13-25.

2 Burks AW, Laubach S, Jones SM. Oral tolerance, food allergy, and immunotherapy: implications for future treatment. J Allergy Clin Immunol 2008; 121:1344-50

3 Branum AM, Lukacs SL. Food allergy among children in the United States. Pediatrics 2009;124:1549-55.

4 Davis KL, Mikita CP. Accidental ingestions in children with peanut allergy. Pediatrics 2007;120:S117.2-8.
5 Greenhawt M. Food allergy quality of life and living with food allergy. Curr Opin Allergy Clin Immunol 2016;16:284-90.

6 Peters RL, Koplin JJ, Dharmage SC, et al. Early exposure to cow's milk protein is associated with a reduced risk of cow's milk allergic outcomes. J Allergy Clin Immunol Pract 2019;7:462-70.

7 Fda approves first drug for treatment of peanut allergy for children. Available: https://www.fda.gov/news-events/press-announcements/fda-approves-firstdrug-treatment-peanut-allergy-children [Accessed 1 May 2020].

8 Cox L, Nelson H, Lockey R, et al. Allergen immunotherapy: a practice parameter third update. J Allergy Clin Immunol 2011;127:S1-55.

9 Trubiano JA, Adkinson NF, Phillips EJ. Penicillin allergy is not necessarily forever. JAMA 2017;318:82-3

10 Vickery BP, Scurlock AM, Kulis M, et al. Sustained unresponsiveness to peanut in subjects who have completed peanut oral immunotherapy. J Allergy Clin Immunol 2014;133:468-75.

11 Wood RA. Food allergen immunotherapy: current status and prospects for the future. J Allergy Clin Immunol 2016;137:973-82.

12 Patriarca C, Romano A, Venuti A, et al. Oral specific hyposensitization in the management of patients allergic to food. Allergol Immunopathol $1984 ; 12: 275-81$.

13 Hofmann AM, Scurlock AM, Jones SM, et al. Safety of a peanut oral immunotherapy protocol in children with peanut allergy. J Allergy Clin Immunol 2009:124:286-91.

14 PALISADE Group of Clinical Investigators, Vickery BP, Vereda A, et al. AR101 oral immunotherapy for peanut allergy. N Engl J Med 2018:379:1991-2001.

15 Chu DK, Wood RA, French S, et al. Oral immunotherapy for peanut allergy (PACE): a systematic review and meta-analysis of efficacy and safety. Lancet 2019;393:2222-32.

16 Grzeskowiak LE, Tao B, Knight E, et al. Adverse events associated with peanut oral immunotherapy in children - a systematic review and meta-analysis. Sci Rep 2020;10:1-12.

17 Lucendo AJ, Arias A, Tenias JM. Relation between eosinophilic esophagitis and oral immunotherapy for food allergy: a systematic review with meta-analysis. Ann Allergy Asthma Immunol 2014;113:624-9.

18 Wright BL, Fernandez-Becker NQ, Kambham N, et al. Baseline gastrointestinal eosinophilia is common in oral immunotherapy subjects with IgE-mediated peanut allergy. Front Immunol 2018:9:2624.

19 Bird JA, Spergel JM, Jones SM, et al. Efficacy and safety of AR101 in oral immunotherapy for peanut allergy: results of ARC001, a randomized, doubleblind, placebo-controlled phase 2 clinical trial. J Allergy Clin Immunol Pract 2018:6:476-85.

20 Approved risk evaluation and mitigation strategies (REMS). Available: https:// www.accessdata.fda.gov/scripts/cder/rems/index.cfm?event=IndvRemsDetails. page\&REMS=398 [Accessed 1 May 2020]

21 FDA Approves Aimmune's PALFORZIATM as First Treatment for Peanut Allergy. Available: http://ir.aimmune.com/news-releases/news-release-details/fdaapproves-aimmunes-palforziatm-first-treatment-peanut-allergy [Accessed 1 May 2020].

22 Gray CL. Current controversies and future prospects for peanut allergy prevention, diagnosis and therapies. J Asthma Allergy 2020;13:51-66. 